\section{MS39-O2 Laser driven acoustic vibrations in nanowires studied by time-resolved GIXD.}

Dmitry Khakhulin ${ }^{1}$, Tomas Stankevic ${ }^{2}$, Peter Vester ${ }^{3}$, Simon O. Mariager ${ }^{4}$, Peter Krogstrup ${ }^{3}$, Michael Wulff ${ }^{5}$, Robert Feidenhans' ${ }^{3}$

1. European XFEL GmbH and the Hamburg Centre for Ultrafast Imaging, Hamburg, Germany

2. MAX IV Laboratory, Lund, Sweden

3. Niels Bohr Institute, Copenhagen, Denmark

4. Paul Scherrer Institute, Villigen, Switzerland

5. European Synchrotron Radiation Facility, Grenoble, France

email: dmitry.khakhulin@xfel.eu

Unique mechanical, thermal and electronic properties of semiconductor nanowires (NW) make them attractive as fundamental building blocks for nanoelectromechanical devices offering extremely high precision for force, mass and strain sensing [1]. Laser driven eigen acoustic vibrations in NWs are defined by their composition, geometry and elastic properties. A single ultrashort laser pulse may simultaneously induce various eigen modes in NWs depending of the excitation conditions.

Acoustic oscillations in nanostructures are usually investigated either by transient optical reflectivity or in case of the slowest modes directly by electron microscopy and stroboscopic imaging. None of these techniques give direct insight into acoustic displacements on the atomic scale. Time-resolved pump-probe $\mathrm{x}$-ray diffraction makes it possible to track the laser induced ultrafast lattice dynamics with great spatial and temporal resolution and has been successfully applied for a number of studies on coherent acoustic phonons in bulk crystals, thin films [2], superlattices [3], and nanostructures [4, 5].

In this contribution we present studies on laser induced acoustic vibrations in crystalline free-standing semiconductor core-shell NWs by time-resolved pump-probe grazing incidence $\mathrm{x}$-ray diffraction (GIXD). Tracking the motion of Bragg peaks on an area detector as a function of the pump-probe delay we were able to record the NWs lattice dynamics with atomic resolution on the sub-nanosecond timescale. Three fundamental acoustic vibrations namely radial breathing, extensional (along the axis) and bending modes generated by a single laser pulse were observed (Fig.1) and characterised. Analysis of the transient lattice displacement magnitudes and frequencies complemented by numeric simulations indicates the dominant mechanism of strain generation and elastic constants of the NWs.

\section{References}

[1] K. Ekinci and M.L. Roukes, Rev. Sci. Instrum., 76, 061101 (2005).

[2] M. Nicoul et al., Appl. Phys. Lett. 98191902 (2011).

[3] M. Woerner et al., Appl. Phys. A 96, 83 (2009).

[4] S.O. Mariager et el., Nano Lett., 10, 2461 (2010).

[5] J. N. Clark et al., Science, 341, 56 (2013).
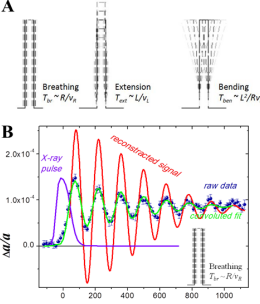

Figure 1. A: the 3 fundamental acoustic vibration modes of a NW; $\mathrm{B}$ : relative lattice displacement measured for the radial breathing mode (blue dots) and de-convoluted signal (red line); C: FEM modeling mesh on the top and the calculated transient strain distribution in the core-shell NW at 130 ps delay.

Keywords: pump-probe diffraction, nanowires, acoustic
C

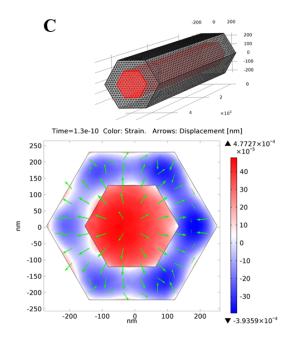

,

(1)

